\title{
Meristem control of leaf patterning
}

\author{
XU Lin \\ National Laboratory of Plant Molecular Genetics, Shanghai Institute of Plant Physiology and Ecology, Shanghai Institutes for Biological \\ Sciences, Chinese Academy of Sciences, Shanghai 200032, China
}

Received January 23, 2015; accepted February 6, 2015

Citation: Xu L. Meristem control of leaf patterning. Sci China Life Sci, 2015, 58: 315-316, doi: 10.1007/s11427-015-4825-4

Plant organs are derived from stem cells. Once a leaf primordium initiates growth from the shoot apical meristem (SAM), it establishes adaxial-abaxial (dorsal-ventral) polarity. This polarity essentially allows the leaf to become a flat structure with the lamina expanding along the juxtaposition, i.e., the middle domain, between the adaxial and abaxial domains. The adaxial domain (upper side) is suitable for capturing light, while the abaxial domain (lower side) is mainly involved in gas exchange [1]. The middle domain, where the vasculature tissues are derived from, is the partition of the adaxial and abaxial domains [2]. Therefore, establishing leaf polarity is not only a classical example of the fundamental question of pattern formation, but is also essential for efficient photosynthesis.

The study of the formation of leaf polarity can be traced back to the 1950s when Sussex performed a series of surgical experiments and established a conceptual framework for the establishment of polarity [3]. Once the connection of the leaf primordium was disrupted from the SAM, the leaf primordium became abaxialized without lamina expansion. Therefore, he proposed the existence of a meristem-derived signal that moves to the adaxial side of the leaf primordium and triggers its adaxial differentiation. In this model, pattern formation of a lateral organ (leaf) is still regulated by the stem cells (SAM) it derives from.

After the first leaf polarity mutant phantastica was described in Antirrhinum majus [4], a group of polarity-controlling genes were identified in different model plants [1]. These genes encode not only transcription factors

email: xulin01@sibs.ac.cn but also microRNAs and trans-acting siRNAs. A wellestablished network of polarity-controlling genes was revealed at the molecular level. Adaxial- and abaxial-controlling genes counteract each other, therefore balancing the two sides of a leaf during its development. However, although the molecular control of leaf polarity has been well studied, the question as to the nature of the moving signal that triggers adaxial establishment remains unresolved. Early studies suggested several candidates including sterol/lipids and small RNAs; however, none of these satisfactorily demonstrated the features of the moving signal.

Recently, a breakthrough in our understanding of the signal mechanism was carried out by Yuling Jiao's lab from the Institute of Genetics and Developmental Biology, Chinese Academy of Sciences, China, and by Elliot Meyerowitz's lab from the California Institute of Technology and Howard Hughes Medical Institute, USA [5]. The present study used real-time confocal observations accompanied by microsurgical experiments; the authors proposed that depletion of auxin from the adaxial domain of the leaf primordium triggers the specification of adaxial identity. Interestingly, in contrast to the original proposal of Sussex that the signal moves from the meristem to the leaf primordium, the auxin signal departs from the primordium to the meristem.

Auxin is a major hormone that has an ability to control cell fate in multiple plant developmental processes. In fact, a study carried out in the late 1990s by the Xu ZhuHong lab at the Shanghai Institute of Plant Physiology, Chinese Academy of Sciences has implied that auxin transport may be involved in leaf polarity patterning [6], although detailed 
mechanism remains unclear. During the very early stage of the establishment of the leaf primordium, auxin was transported into cells that initiate the primordium. The present study [5] found that once a leaf primordium emerges from the SAM, auxin near the adaxial domain was transported back to the SAM, thereby forming low and high auxin concentrations in the adaxial and abaxial domains, respectively. The gradient of auxin distribution establishes the adaxial-abaxial polarity. Therefore, either adding exogenous auxin in the adaxial domain of a leaf primordium or blocking the polar transportation of auxin from the adaxial domain to the meristem resulted in abaxialized leaf development. In addition, the authors showed that the auxin signaling pathway gene MONOPTEROS $(M P)$ was involved in the process in response to auxin depletion. $M P$ is an AUXIN RESPONSE FACTOR family gene, which is directly involved in the auxin signaling pathway. Its expression was identified in the adaxial domain, where the auxin was depleted, suggesting that the function of MP may be primarily involved in the middle domain.

This study provides the first evidence of the identification of the proposed signal. Following this study, the identi- fication of the molecular network that links the auxin signaling to leaf-polarity-controlling genes will further improve our understanding of the early molecular events that occur during leaf polarity establishment. In addition, analysis of the role of the middle domain during the early stage of polarity formation may also provide novel insights into the field of axial patterning.

1 Husbands AY, Chitwood DH, Plavskin Y. Signals and prepatterns: new insights into organ polarity in plants. Genes Dev, 2009, 23: 1986-1997

2 Nakata M, Matsumoto N, Tsugeki R, Rikirsch E, Laux T, Okada K. Roles of the middle domain-specific WUSCHEL-RELATED HOMEOBOX genes in early development of leaves in Arabidopsis. Plant Cell, 2012, 24: 519-535

3 Sussex IM. Experiments on the cause of dorsiventrality in leaves. Nature, 1951, 167: 651-652

4 Waites R, Hudson A. Phantastica: a gene required for dorsoventrality of leaves in Antirrhinum majus. Development, 1995, 121: 2143-2154

5 Qi J, Wang Y, Yu T, Cunha A, Wu B, Vernoux T, Meyerowitz E, Jiao Y. Auxin depletion from leaf primordia contributes to organ patterning. Proc Natl Acad Sci USA, 2014, 111: 18769-18774

6 Ni DA, Wang LJ, Xu ZH, Xia ZA. Foliar modifications induced by inhibition of polar transport of auxin. Cell Res, 1999, 9: 27-35

Open Access This article is distributed under the terms of the Creative Commons Attribution License which permits any use, distribution, and reproduction in any medium, provided the original author(s) and source are credited. 